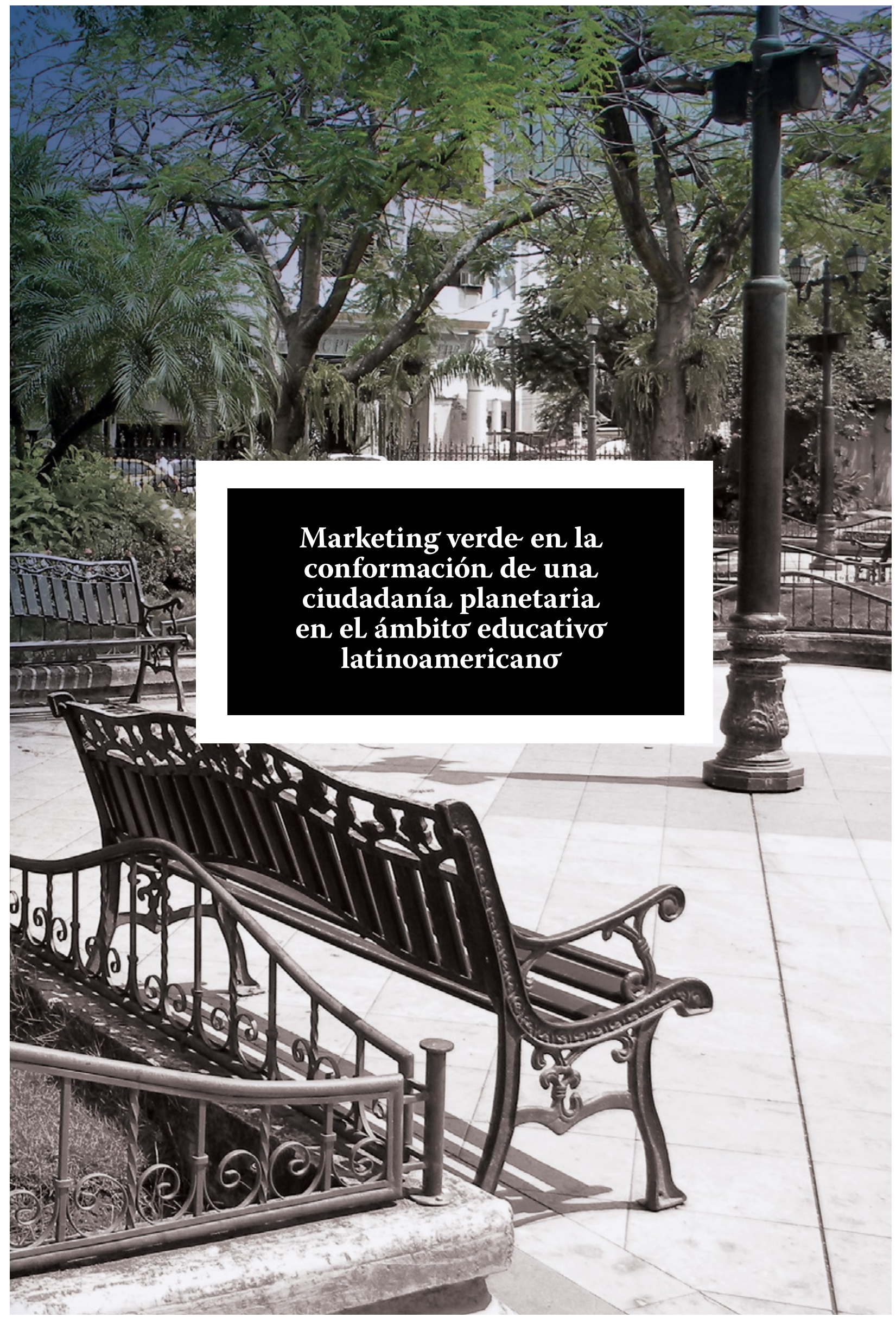




\section{MARKETING VERDE EN LA CONFORMACIÓN DE UNA CIUDADANía PLANETARIA EN EL ÁMBITO EDUCATIVO LATINOAMERICANO}

\section{GREEN MARKETING IN THE CONFORMATION OF A PLANETARY CITIZENSHIP IN THE EDUCATIONAL FIELD LATINAMERICAN}

\section{RESUMEN}

El artículo de revisión tuvo por objetivo analizar el aporte del marketing verde en la conformación de una ciudadanía planetaria en el ámbito educativo latinoamericano. Se empleó una metodología de tipo documental con un diseño bibliográfico que permitió estructurar la información recopilada, se aplicó un análisis de contenido con la finalidad de descubrir las competencias implícitas en la información analizada. Entre las principales conclusiones se tiene que el marketing verde ofrece a la educación, la posibilidad de edificar competencias en el estudiante en pro de configurar un pensamiento global, el cual debe ser fomentado desde una educación verde planetaria latinoamericana para el contexto global.

PALABRAS CLAVE: Ecología humana; educación ambiental; educación cívica; ética ambiental; investigación centrada en un problema.

Copyright @ Revista San Gregorio 2019. ISSN 1390-7247; eISSN: 2528-7907 @

\section{ABSTRACT}

The article of revision its objective was to analyze the contribution of green marketing in the formation of a planetary citizenship in the Latin American educational environment. A methodology was used of documentary type with a bibliographic design; a content analysis was applied in order to discover the implicit competences in the information analyzed. Among the main conclusions is that green marketing offers education the possibility of building competences in the student in favor of configuring a global thinking, which should be encouraged from an education Latin American planetary green for the global context.

KEYWORDS: Human ecology; Environmental education; Civic education; environmental ethics; Problem focused research.

Copyright @ Revista San Gregorio 2019. ISSN 1390-7247; eISSN: 2528-7907 @

\section{Julio Juvenal Aldana Zavala}

Universidad Nacional Experimental Francisco de Miranda (UNEFM).

Falcón. Venezuela.

$\sim$

julioaldanazavala@gmail.com
Félix Colina Ysea

A

Universidad SISE. Lima, Peru

- fcolina@universidadsise.edu.pe 


\section{INTRODUCCIÓN}

La ciudadanía planetaria es una concepción en procura que las personas desarrollen un modo de pensar que les permita interconectar las diversas realidades que le rodean con el fin de gestionar la reflexibilidad en favor de analizar el contexto social como una red en donde cada componente del sistema influye en el devenir de las acciones sociales, en este sentido, la Organización de las Naciones Unidas (ONU), asume esta interconectividad en su declaratoria sobre los Objetivos de Desarrollo Sostenible (ODS), "conformada por 17 objetivos que deben ser alcanzados en los próximos años, específicamente de cara al 2030" (ONU, 2015 , p. 1) destacándose que en el fondo esta declaratoria procura la protección y preservación del medio ambiente por medio de conductas sostenibles.

Es así que la educación tanto formal e informal en todos los niveles de la vida juega un papel primordial en lograr este acometido, asumiendo la Organización de las Naciones Unidas para la Educación, la Ciencia y la Cultura (UNESCO), su agenda Incheon 2015 "una educación inclusiva y de calidad a lo largo de la vida", siendo además el organismo encargado de desarrollar el objetivo 4 de los ODS, concatenándose la posibilidad de articular políticas educativas favorecedoras de un pensamiento reflexivo complejo en concordancia con la protección del medio ambiente, siendo así que surge la visión de una ciudadanía planetaria en donde cada actor social desde su glocalidad, debe asumir una postura de respeto no solo para sus semejantes, sino, para el medio terrenal donde se desenvuelve.

Es allí donde entra el marketing verde como una alternativa generadora de un pensamiento global planetario desde el respeto por el medio ambiente, situación que la educación formal e informal, podría asumir como una estrategia permanente para generar un aprendizaje de calidad a lo largo de la vida, arti- culándose la gestación de un ciudadano con visión planetaria, para esto es indispensable transcender la visión del marketing verde como una opción meramente comercial, para asumirla como una filosofía que viene a complementar la formación académica - cultural de la sociedad en función que esta asuma en cada momento de su vida una interacción de respeto por la vida, en especial del medio ambiente, siendo así, un pensamiento complejo desde lo ecológico.

Generándose una interrelación entre lo educativo, cultural, económico, ambiental, mediante la cual, el ser humano se articule como agente transformador, liberador, y no dominador, explotador, del medio ambiente por el mero detrimento del mismo, para esto (Murga-Menoyo, 2018, p. 49) plantea que se "exige a la educación un enfoque metodológico que permita abordar las cuestiones desde perspectivas múltiples, contando para ello con el concurso de las diferentes disciplinas". Esto implica generar transformaciones curriculares desde donde se asuma la conexión que tiene la formación de un ciudadano con conciencia planetaria, siendo necesario incluir lo relacionado al marketing verde como una filosofía ecológica que permita trabajar en un aprendizaje que transcienda los escenarios escolares para constituir el mundo en una gran aula abierta. En complemento de lo expuesto (Murga - Menoyo y Novo, 2017) colaboran con:

Un planteamiento «glocal» conduce, en nuestra opinión, hacia una comprensión amplia del concepto de ciudadanía, que toma en cuenta no sólo el sentido de pertenencia de las personas respecto de sus contextos, sino también los límites y necesidades del planeta en su condición de «casa común» de todos los humanos (p. 57).

Es necesario redescubrir la educación y fomentar la visión planetaria desde una ciudadanía que respete, valore, lo ambiental, ecológico, lo cual además podría influir en la configuración de múltiples valores en el ser humano, por cuanto quien valora su entorno social - ambiental, ha alcanzado una conciencia ciudadana favorecedora de una convivencia intercultural en proximidad a una cosmovisión compleja de ser en convivencia con el otro y el medio ambiental. En ese sentido, (Moreno-Fernández \& Navarro-Díaz, 2015, p. 175) complementan la idea al indi- 
car que "Una educación ambiental que debe promover la adquisición de una conciencia, valores y comportamientos que beneficien la participación eficiente de la ciudadanía en el proceso de toma de decisiones con respecto al entorno en el que se vive". Siendo oportuno desde una educación basada en la resolución de problemas, ejemplificar estrategias desde el marketing verde adaptadas didácticamente a la educación, una posible aliada para generar, además, la innovación, creatividad, reflexibilidad de los estudiantes.

En complemento de lo expuesto, (Ottman, 2006 como se citó en Monteiro y colaboradores, 2015, p. 112) plantea “...que la primera regla del marketing tradicional y del marketing verde es enfocarse en la presentación de los beneficios a los clientes". Los estudiantes deben ser percibidos como clientes del mundo donde habitan, para lo cual es necesario fomentar su conciencia hacia la valoración de lo ambiental - ecológico, siendo así que el marketing verde debe ser adaptado como estrategia didáctica a los diversos niveles educativos, articulándose una publicidad desde lo educativo para la conformación de un ciudadano planetario, así la educación redescubre su esencia de formar para la vida y no solo a una persona capacitada con contenidos curriculares.

Existiendo así la posibilidad que la educación en todos sus niveles educativos, incluyendo el universitario, adopte una cosmovisión planetaria global desde lo ecológico, siendo que la educación basada en problemas permite trabajar además en resolución de proyectos que pueden fomentar el aprendizaje de calidad a lo largo de la vida, dado que su esencia se basa en la investigación, siendo así posible configurar un aprendizaje que transcienda el modelo transmisionista de conocimientos, por uno generador de conocimientos, por consiguiente en el actual artículo de revisión, se usa una metodología documental para el análisis e interpretación de documentos investigativos que proyectan la necesidad de promover una educación significativa en donde lo ecológico y ambiental se encuentre presente en el devenir educativo, de ahí que surge la interrogante

¿Cuál es el aporte del marketing verde en la conformación de una ciudadanía planetaria en el ámbito educativo latinoamericano?

\section{OBJETIVO DE LA INVESTIGACIÓN}

Analizar el aporte del marketing verde en la conformación de una ciudadanía planetaria en el ámbito educativo latinoamericano.

\section{METODOLOGÍA}

Se trabajó con una investigación documental (Palella y Martins, 2012, p. 90) señalan que "Se concreta exclusivamente en la recopilación de información en diversas fuentes. Indaga sobre un tema en documentos escritos $\mathrm{u}$ orales; uno de los ejemplos más típicos de este tipo de investigación son las obras de historia”. En cuanto al diseño de la investigación se refiere al bibliográfico, explicado por (Palella y Martins, 2012) como aquel que:

Se fundamenta en la revisión sistemática, rigurosa y profunda de material documental de cualquier clase. Se procura el análisis de los fenómenos o el establecimiento de la relación entre dos o más variables. Cuando opta por este tipo de estudio, el investigador utiliza documentos; los recolecta, selecciona, analiza y presenta resultados coherentes. El diseño bibliográfico utiliza los procedimientos lógicos y mentales propios de toda investigación: análisis, síntesis, deducción, inducción, entre otros (p. 87).

En función de lo planteado, se abordó desde una vertiente teórica las variables marketing verde y ciudadanía planetaria como elementos centrales para generar un análisis reflexivo que proponga una alternativa pedagógica - didáctica en el ámbito educativo latinoamericano con la finalidad de contribuir en la articulación de aprendizajes de calidad a lo largo de la vida, en función de interconectar al estudiante con su realidad global mediante la valoración del respeto al medio ambiente, viéndose implícitos valores interculturales con sus semejantes, para lo cual fue necesario recurrir a una muestra poblacional conformada por artículos de investigación de revistas arbitradas e indizadas, así como libros relacionados a la metodología de la investigación, usándose el análisis de contenido como técnica analítica de la información recopilada.

\section{RESULTADOS}

En función de la opinión y aporte de autores, se presentan dos tablas sinópticas, una relacionada al marketing verde y la siguiente a ciudadanía planetaria, con la finalidad de generar un análisis de contenido y así, esta- 
blecer resultados que permitan concatenar una postura analítica de salida por parte de los investigadores, en ese sentido:

TABLA 1. MARKETING VERDE

Autor Cita
Análisis por parte de investigadores
Monteiro y Colaboradores (2015).

El marketing verde es un concepto relativamente nuevo para la mayoría de los consumidores (p. 120).

En relación con los recursos que se utilizan para representar al

Sabre (2014). medioambiente en los mensajes, se destacan aquellos visuales frente a los verbales (p. 36).

Echeverri (2010).

Del Campo y González (2010)

Escobar Moreno, (2015).

Cardona

Arbeláez. (2018).
El compromiso hoy es desarrollar un acercamiento con el que se proteja la naturaleza (p. 137).

Color verde que es "típicamente ecológico por su asociación con el color de la vegetación"

Puede afirmarse que los consumidores verdes de la región estudiada, son personas a las que les gusta estar actualizadas y que tienen una alta atracción por la tecnología (p. 106).

Innovar no es hacer pequeños cambios en un producto o servicio ya ofrecido al público, más bien consiste en crear
La concepción verde no solo implica la venta de un producto, sino, una filosofía de vida que permite integrar el accionar ciudadano con el cuidado del medio ambiente.

El estudiante podría diseñar estrategias comunicativas que le permitan comunicar el valor que tiene el medio ambiente para la humanidad, fomentándose su innovación y proactividad.

Ser productivo desde lo sostenible implica una relación ganar - ganar entre el ser humano y el medio ambiente, fomentándose así, una cultura responsable.

El estereotipo verde podría ser un factor de partida para la articulación de acciones que permitan tener presente en el ambiente de aprendizaje, lo ambiental - ecológico como una apuesta concientizadora de los estudiantes en consecución de una conciencia planetaria.

Fomentar el gusto por el respeto al medio ambiente, podría contribuir en la formación de investigadores, por cuanto se concibe una personalidad indagadora de los productos a consumir, mediante el uso de las TIC, situación que podría contribuir en un aprendizaje de calidad.

La innovación se encuentra presente en la filosofía verde, de allí que puede ser transferida a la generación de un aprendizaje 
TABLA 1. MARKETING VERDE (CONT.)

\begin{tabular}{ll} 
Autor & Cita \\
\hline Díaz (2008). $\quad \begin{array}{l}\text { Las actividades de ecoturismo tienen que } \\
\text { estar acompañadas permanentemente de } \\
\text { tareas de investigación (p. 148). }\end{array}$
\end{tabular}

de Tendencia

Futuro $360^{\circ}$

(2017).

Bur (2013)

Pérez,

Espinoza y

Peralta

(2016) Todas las estrategias implementadas para reducir el impacto sobre el medio ambiente son positivas, pero deben partir de una fuerte ética empresarial, que permitan la generación de medidas efectivas con el medio ambiente a partir de la voluntad y la decisión del empresario (p. 109).

El marketing sustentable dejó de ser una alternativa para transformarse en una necesidad para las empresas (p. 140).

No importa el tamaño ni la naturaleza de la empresa, lo que realmente importa es que se tome conciencia a todo nivel, desde la alta dirección hasta los más bajos niveles organizacionales de que la tierra es la que provee de todos los recursos que se utilizan para la
Análisis por parte de investigadores

El estudiante podría aprender haciendo investigación, esto mediante la aplicación de técnicas de recolección y análisis de información, desde la recogida de datos en contextos naturales, existiendo contacto directo con el fenómeno de estudio, existiendo un aprendizaje en contexto.

La preservación del medio producción, por tanto, se debe cultivar para que las futuras generaciones disfruten de ella (p. 176).

La conservación del medio ambiente es muy importante y requiere del trabajo en conjunto del Estado, organizaciones y consumidores (p. 163). ambiente trae implícito el fomento de la ética, la cual permite al ser humano reflexionar sobre el bien y mal que pueda cometer, en este caso con el medio ambiente, siendo posible la generación de una competencia ética desde los ambientes de aprendizaje.

Lo sustentable es una tendencia en crecimiento, lo cual los estudiantes deben estar preparados para asumir los retos de la sociedad global.

Generar el sentido de pertenencia por la tierra es un factor generador de una ciudadanía con conciencia, además agradecida de vivir en un entorno sano, el cual debe ser un legado a las próximas generaciones, formándose así a los estudiantes en el valor de la co responsabilidad.

La integración escuela comunidad, puede ser fomentada desde la filosofia verde, además desde ese binomio se puede articular una participación ciudadana comprometida con el diseño de políticas educativas cónsonas a la realidad global. 
Autor

Cita

En cuanto al sentimiento de pertenecer a una comunidad global, que el alumnado se sienta parte de una ciudadanía

Moreno

(2015)

Feldmann y

Biderman

(2001)

Abril (2015)

Muñoz

(2014)

Gómez

(2016) planetaria, aunque no entienda el concepto a grandes rasgos, y considere que se debe de actuar desde la solidaridad, es un paso importante para poder comenzar a trabajar desde la escuela (p. 55).

Estamos frente a un problema cuya solución implica transformaciones reales del actual paradigma de desarrollo. Depende de la formación de una ciudadanía planetaria, de ciudadanos preocupados y comprometidos con el futuro de la vida en la Tierra (p. 292).

Cuando frente a la crisis de representatividad se apela a "los políticos" como culpables de buena parte de los males que aquejan a la sociedad, tal y como sostiene buena parte de la opinión pública, la escuela y quienes nos dedicamos a la educación también deberíamos sentirnos interpelados ( $\mathrm{p}$. 103).

Las políticas públicas y las normas para la defensa de los derechos humanos fundamentales como principio cosmopolita demandan, antes de nada, de la formación en el carácter de cada ser humano en lo socialmente bueno (p. 203).

Mientras la participación, la interculturalidad, la ecología siguen siendo materias de malla curricular "la calle" revierte procesos intelectualizados. La calle está interculturalizando, dialoga con la naturaleza y genera un nuevo contrato socioeconómico ambiental (p. 88).

\section{Análisis por parte de} investigadores

\begin{abstract}
La solidaridad se encuentra inmersa en la articulación de un ciudadano planetario, por consiguiente, la escuela debe plantearse asumir la fusión con lo verde para construir una visión en donde la sinergia con el otro conforme equipos de trabajos cooperativos bajo la visión ganar - ganar.
\end{abstract}

La educación actual es responsable de promover un estilo de vida sustentable, lo cual representa formar a un ciudadano comprometido con la transformación social, mediante la preservación del medio ambiente.

Es necesario asumir la responsabilidad de ser un ciudadano en el mundo, lo cual implica tener participación activa en la construcción de una mejor sociedad, para lo cual es indispensable que las personas se organicen para asumir una participación ciudadana comprometida.

Desde el trabajo por la defensa y promoción de los derechos humanos, se asume la preservación del medio ambiente, siendo este un elemento a tener en cuenta en la formación de ciudadanos planetarios desde la escuela.

Es necesario transcender el mero estudio cognitivo de lo ecológico, ambiental, intercultural, es pertinente la formación práctica para la consecución de una verdadera conciencia planetaria. 
TABLA 2. CIUdAdANía PLANETARIA (CONT.)

Autor

Cita
Análisis por parte de investigadores

\begin{tabular}{|c|c|}
\hline $\begin{array}{l}\text { Zambrano } \\
(2015)\end{array}$ & $\begin{array}{l}\text { La mayoría de docentes no utilizan } \\
\text { estrategias adecuadas para orientar la } \\
\text { educación ambiental en los niños y niñas, } \\
\text { los recursos didácticos que utilizan no } \\
\text { están en función de los hábitos para el } \\
\text { cuidado ambiental (p. 20). }\end{array}$ \\
\hline $\begin{array}{l}\text { Hernández y } \\
\text { López (2014) }\end{array}$ & $\begin{array}{l}\text { Así el desafio de las universidades, tanto } \\
\text { públicas como privadas hoy, será lograr } \\
\text { un modelo universitario centrado en la } \\
\text { formación del ciudadano, en el cual la } \\
\text { construcción de la democracia y de la } \\
\text { participación social sea la que alimente la } \\
\text { nueva forma de vernos y de relacionarnos } \\
\text { con otros y con el mundo que nos rodea } \\
\text { (p. 55). }\end{array}$ \\
\hline
\end{tabular}

Ceballos \&

Ocampo

(2016)

Collado

(2018)

Aranibar

(2010)

Castillo y

Castillo

(2013)

De Souza

(2014)
Unida al Pensamiento Complejo la

Responsabilidad Social alcanzará su máximo desarrollo (p. 75).

Tenemos que aprovechar la oportunidad que nos brindan los ODS para caminar juntos hacia un horizonte civilizatorio sostenible con el medio ambiente (p. 82).

La no fragmentación de los saberes, la reflexión sobre lo que se enseña y la elaboración de un paradigma de relación circular entre las partes y el todo, lo simple y lo complejo (78).

Hoy más que nunca se reconoce que lo afectivo es un imperativo en el que tiene que fundamentarse la biopedagogía, es la esperanza de lograr aprendizajes más sociales, humanos, vitales; eso concatena

Merece destacarse el hecho de que el desarrollo sustentable posee como objetivo principal la promoción del bienestar de las personas sin degradar el capital natural

(p. 239)
Es pertinente integrar estrategias adecuadas e innovadoras a la formación de la responsabilidad ciudadana ambiental, siendo esto posible por medio del marketing verde.

Las universidades están llamadas a formar profesionales comprometidos con la articulación de una sociedad sostenible y sustentable, para lo cual, la filosofía verde puede ser un punto de referencia para fomentar la conciencia necesaria en los estudiantes para asumir tal rol protagónico.

Es necesario promover el aprendizaje complejo, sistémico, complementario, dejando de lado la enseñanza atomizada de conocimientos.

Existe la voluntad política internacional por lo sostenible, de allí la urgencia de trabajar desde la filosofia verde en la consecución de una ciudadanía apta para asumir tal reto.

No existen saberes ni conocimiento menos importante que otro, hoy es necesario ser interdisciplinario y concatenar la experiencia con lo científico, para construir conocimientos pertinentes a las necesidades reales de la sociedad global.

La biopedagogía aflora como una aliada en el proceso de concatenar el marketing verde con la gestación de un ciudadano planetario.

Se trata de crear una conciencia colectiva de aprovechamiento de los recursos naturales sin destruir los mismos, situación que puede ser desde un ciudadano ético, responsable y con conciencia planetaria, tarea de la educación en todos sus niveles. 


\section{DISCUSIÓN}

Desde las matrices de análisis, se estructuran categorías que permiten articular los aportes de las variables de estudio al cumplimiento del objetivo de investigación, así mismo, permite establecer una interconexión entre ambas para generar el aporte a la educación latinoamericana, en este sentido se presenta la Figura 1 (Ver en Anexos).

El marketing verde debe ser comprendido como una filosofía de vida que permite al ser humano generar estrategias comunicativas asertivas y efectivas con la finalidad de relacionarse con su entorno social, mediante una aptitud innovadora y proactiva que promueve el emprendimiento empresarial desde una visión productiva sostenible, esto con la intención de crear una cultura responsable sobre el uso de los recursos procesados para la obtención de bienes y servicios, los cuales deben contener implícitos el respeto a lo ambiental ecológico, estando así en posibilidad de transcender los retos globales del mundo actual y venidero donde la contaminación, perdida de animales, vegetación, son parte de los compromisos ineludibles que el ser humano enfrentará de no asumir con responsabilidad su relación con lo ambiental - ecológico.

La escuela - comunidad juega un papel primordial por cuanto es eslabón primario donde se forma al ciudadano, para lo cual se requiere una participación protagónica en la elaboración de políticas públicas que apunten a la sostenibilidad del mundo. El uso sostenible de las TIC, permite educar para la vida, brindando pasos para aprender haciendo investigación, lo cual implica estar inmersos en la praxis investigativa de contacto directo entre los estudiantes y el medio ambiente, de ese modo, se forma el carácter ético de la persona, situación que le permitirá discernir entre el bien y mal, lo conveniente para actuar bien en pro del cuido ambiental, bridándose pasos para la consecución de una ciudadanía planetaria al servicio de la humanidad.

\section{CONCLUSIONES}

Es necesario educar con una visión de ciudadanía planetaria, esto implica redescubrir la esencia de la educación, siendo esta educar y formar para la humanidad, para la vida, siendo indispensable transcender el conocimiento fragmentado, mecanicista, por un aprendizaje fomentado desde la investigación, lo cual permitirá contar con investigadores críticos, reflexivos, éticos, que comprendan que el conocimiento se actualiza en la medida que se obtienen nuevos resultados investigativos;

Además, el contacto con la naturaleza y contexto social, permitirá a los estudiantes aplicar técnicas de recolección de datos in situ, siendo así una oportunidad de aprender haciendo, lo cual implica fomentar la conexión del ser con el medio global que le rodea, estrategia que podría favorecer el paso de la educación a un enfoque que permita promover un aprendizaje complejo;

Al interconectar la filosofía verde con la educación, se fomenta una ciudadanía planetaria en pro de la formación de competencias comunicativas, investigativas, éticas, tecnológicas, innovadoras, emprendedoras, entre otras, con lo cual se promueve un paradigma sustentable de producción que procura transcender el modelo rentable del asalariado, es decir, que implícitamente se estaría educando en la formación de emprendedores que promuevan una economía basada en lo sostenible.

Latinoamérica juega un papel preponderante en el futuro global de las próximas décadas, es necesario promover una nueva visión mundo donde el respeto por el medio sea factor primordial para la elaboración de acciones que permitan un mejor vivir al ser humano en sana convivencia con sus semejantes y medio ecológico donde habitan, entendiendo que la globalidad no solo es tecnológica y comunicativa, sino, ambiental, siendo el mundo la gran casa donde habitamos y debemos preservar;

En resumen el marketing verde ofrece a la educación, la posibilidad de contar con una filosofía que promueve lo ambiental - ecológico en un marco de respeto, situación que permite edificar competencias en el estudiante en pro de configurar un pensamiento global en razón de comprender la importancia de ser un ciudadano planetario para la vida $y$ sostenimiento de la tierra como proveedora de los recursos necesarios para el crecimiento humano, concepción que debe ser fomentada desde una educación verde planetaria latinoamericana para el contexto global. 


\section{REFERENCIAS BIBLIOGRÁFICAS}

Abril Hervás, D. (2015). Ciudadanía, educación y complejidad: miradas desde la ecopedagogía. Diálogo andino, (47), 93-103. https://dx.doi.org/10.4067/S0719 26812015000200010

Aranibar Brañez, D. (2010). Los siete saberes según Edgar Morín. Gaceta Médica Boliviana, 33(1), 76-78.

Bur, A. (2013). Marketing sustentable. Utilización de marketing sustentable en la industria textil y de la indumentaria. Recuperado de https://dialnet.unirioja.es/ descarga/articulo/4200817.pdf

Cardona Arbeláez, D. (2018). Marketing Green y el avance tecnológico como apuesta de innovación para el desarrollo de las estrategias de marketing. Recuperado de http://www.revistaespacios.com/a18v39n26/ a18v39n26p02.pdf

Castillo, I. y Castillo, R. (2013). La mediación biopedagógica desde una perspectiva ética. Recuperado de http://www.revistas.una.ac.cr/index.php/EDUCARE/ issue/current

Ceballos Betancur, W., \& Ocampo Suárez, H. (2016). Hacia una Responsabilidad Social Universitaria sistémico-compleja. Una lectura desde el pensamiento de Edgar Morin. Recuperado a partir de http://ojs.tdea.edu. co/index.php/senderos/article/view/396

Collado Ruano, J. (2018). El paradigma de la cosmodernidad: reflexiones filosóficas sobre ciencia religión. Recuperado de https://dx.doi.org/10.17163/ soph.n24.2018.01

De Souza Bonfim, Cristiane Ingrid. (2014). Educación ambiental para la preservación de la vida. Recuperado de http://www.scielo.org. $\mathrm{mx} /$ scielo.php?script=sci

arttext\&pid=S2007-81102014000200008\&lng=es\&tlng= es.

Del Campo, S., y González, R. (2010). Referencias al medio ambiente en los mensajes publicitarios. Investigación de la publicidad en España entre 2006 y 2007. Recuperado de http://adresearch.esic.edu files/2010/10/Texto-completo-PDF-aDResearch-ESICvol.-2-De-Andr\%C3\%A9s-y-Gonz\%C3\%A1lez-2010.pdf

Díaz, R. (2008). Marketing ecológico y turismo Recuperado de http://www.scielo.org.ar/pdf/eypt/v17n2/ v17n2a04.pdf

Echeverri, L. (2010). Inserción del mercadeo verde en prácticas empresariales en Colombia (Casos De Estudio) Recuperado de http://www.scielo.org.co/pdf/luaz/n31/ n31a09.pdf

Escobar-Moreno, N. (2015). Caracterización preliminar del consumidor verde antioqueño: El caso de los consumidores del Valle de Aburrá. Recuperado de http:// www.scielo.org.co/pdf/ean/n78/n78a07.pdf

Feldmann, F., y Biderman, R. (2001). Los cambios climáticos globales y el desafío de la ciudadanía planetaria. Recuperado de https://scielo.conicyt.cl/pdf/ abioeth/v7n2/art10.pdf

Gómez Abeledo, G. (2016). Medioambientalismo Interculturalidad. SOS PANADEIRA "Muerte del patrón". Recuperado de http://revista.sangregorio.edu.ec/index. php/REVISTASANGREGORIO/article/view/258

Hernández Flores, H., \& López Calva, J. (2014). La participación estudiantil como estrategia de formación ciudadana para la educación en valores en el nive superior. Recuperado de doi:10.15198/ seeci.2014.35E.43-58
Monteiro, A., y Colaboradores (2015). Mezcla del marketing verde: una perspectiva teórica. Recuperado de https://www.redalyc.org/pdf/462/46243484005.pdf

Moreno-Fernández, O., \& Navarro-Díaz, M. (2015). Educación ambiental, ciudadanía y participación. Recuperado de https://www.upo.es/revistas/index.php/ IJERI/article/view/1470

Moreno Fernández, O. (2015). Educación y ciudadanía planetaria. Concepciones del alumnado participante en programas educativos andaluces. Recuperado de https: / dialnet.unirioja.es/descarga/articulo/5099321.pdf

Murga-Menoyo, M. (2018). La Formación de la Ciudadanía en el Marco de la Agenda 2030 y la Justicia Ambiental. Recuperado de doi: http://dx.doi. org/10.15366/riejs2018.7.1.002

Murga - Menoyo, M., y Novo, M. (2017). Sostenibilidad, desarrollo «glocal» y ciudadanía planetaria. Referentes de una Pedagogía para el desarrollo sostenible. Recuperado de doi:10.14201/teoredu2915578

Muñoz Cardona, Á. (2014). La ciudadanía: un deber planetario. Recuperado de https://revistas.udem.edu.co/ index.php/economico/article/view/938

Observatorio de Tendencia Futuro $360^{\circ}$ (2017). Economía verde y ecoeficiencia: paradigmas para la sostenibilidad. Recuperado de http://revistas.esumer.edu.co/index.php/ merc/article/view/142

Ottman, J.A. (2006). The Rules of "Green" Marketing. Citado por Monteiro, A. (2015). Mezcla del marketing verde: una perspectiva teórica. Recuperado de https:// www.redalyc.org/pdf/462/46243484005.pdf

Organización de las Naciones Unidas (2015). Objetivos de desarrollo sostenible. Recuperado de https://www. un.org/sustainabledevelopment/es/objetivos-dedesarrollo-sostenible

Palella Stracuzzi, Santa y Martins Pestana, Feliberto. (2012). Metodología de la investigación cuantitativa Fondo editorial de la Universidad Pedagógica Libertador. Caracas, Venezuela.

Pérez Espinoza, J.M., Espinoza Carrión, C., \& Peralta Mocha, B. (2016). La responsabilidad social empresarial y su en-foque ambiental: una visión sostenible a futuro. Recuperado de http://scielo.sld.cu/pdf/rus/v8n3/ rus23316.pdf

Sabre, M.E. (2014). La publicidad verde en Argentina. Análisis del uso de apelaciones medioambientales en anuncios televisivos. Recuperado de http://www. cuadernos.info/index.php/CDI/article/view/cdi.34.544

Salas Canales, H. (2018). Marketing ecológico: La creciente preocupación empresarial por la protección de medio ambiente. Recuperado de http://www. revistasbolivianas.org.bo/pdf/rfer/v15n15/v15n15 a10. pdf

UNESCO (2015). Educación 2030: Declaración de Incheon y Marco de Acción para la realización de Objetivo de Desarrollo Sostenible 4. Recuperado de https://unesdoc.unesco.org/ark:/48223/pf0000245656 spa

Zambrano Montes, L. (2015). Formación de hábitos para el cuidado del medio ambiente en la educación inicial. Recuperado de http://revista.sangregorio.edu.ec/index. php/REVISTASANGREGORIO/article/view/53/9 


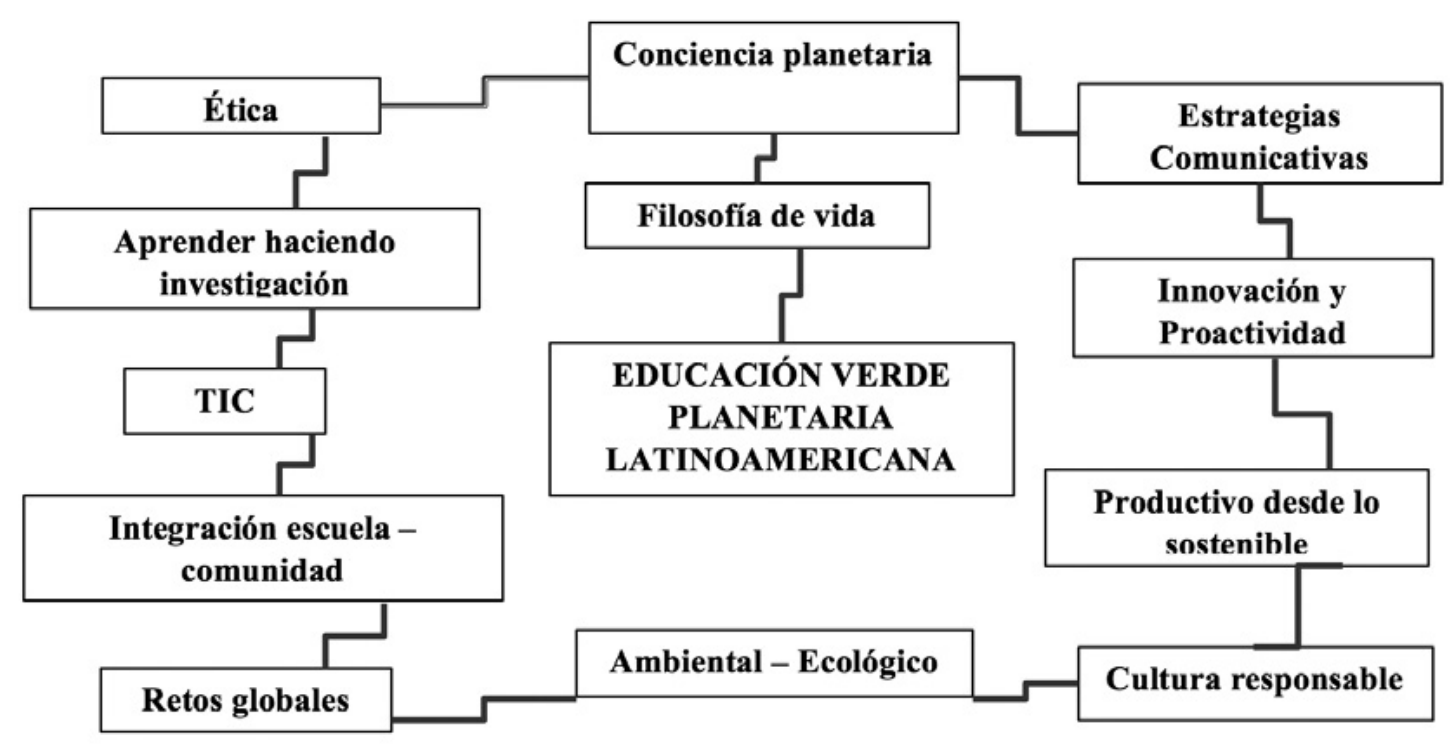

Figura 1. Educación Verde Planetaria Latinoamericana.

Fuente: Elaborado por investigadores. 


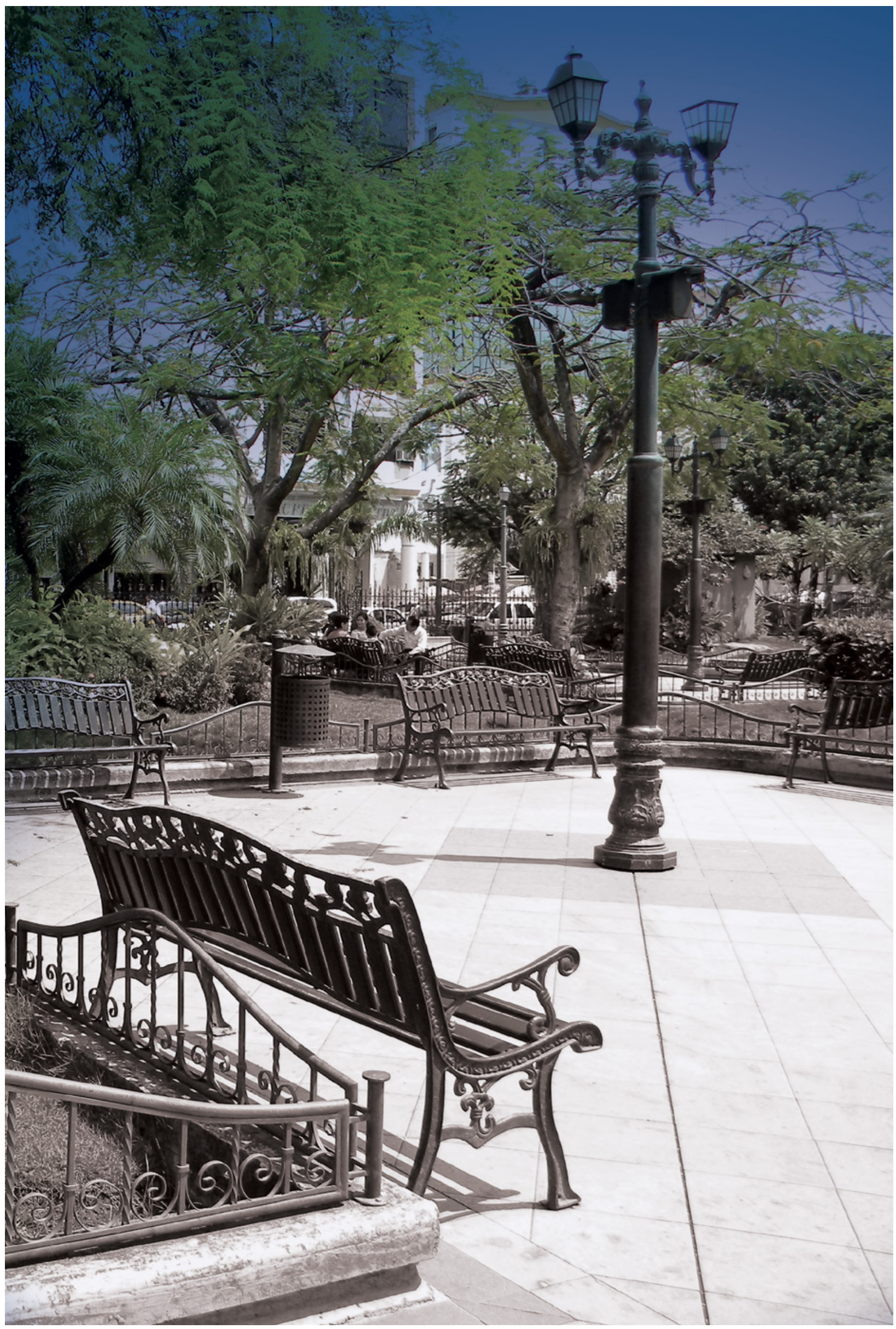

